\title{
TECNICA DI RICAMO IN LINEA PER LO SVILUPPO CREATIVO E IL MOTORE APPLICABILE NELLE CLASSI DI EDUCAZIONE ARTISTICA DELLE SCUOLE SUPERIORI
}

\section{ARTICOLO ORIGINALE}

DUTRA, Ana Paula de Souza1, FECURY, Amanda Alves², DENDASCK, Carla Viana ${ }^{3}$, OLIVEIRA, Euzébio de ${ }^{4}$, DIAS, Claudio Alberto Gellis de Mattos ${ }^{5}$

DUTRA, Ana Paula de Souza. Et. Tecnica di ricamo in linea per lo sviluppo creativo e il motore applicabile nelle classi di educazione artistica delle scuole superiori. Revista Científica Multidisciplinar Núcleo do Conhecimento. Anno 06, Ed. 01, Vol. 08, pp. 26-33. gennaio 2021. ISSN: 2448-0959, Link di accesso: https://www.nucleodoconhecimento.com.br/formazione-it/tecnica-del-ricamo, DOI: 10.32749/nucleodoconhecimento.com.br/formazione-it/tecnica-di-ricamo

\section{RIEPILOGO}

L'educazione artistica è composta da diverse tecniche che portano allo sviluppo del pensiero logico, della creatività e del coordinamento motorio. Lo scopo di questo lavoro è quello di descrivere una tecnica di ricamo in linea per lo sviluppo creativo e il motore applicabile nelle classi di educazione artistica delle scuole superiori. II metodo descritto sarà il ricamo gratuito : immagini, una tecnica che utilizza tessuto,

\footnotetext{
${ }^{1}$ Laureato in Belle Arti (FAAP SP), studia Specializzazione in Arti, Rudolf Steiner College (FRS SP).

${ }^{2}$ Biomedicale, Dottorato di Ricerca in Malattie Tropiche, Professore e ricercatore del Corso di Medicina del Campus Macapá, Università Federale di Amapá (UNIFAP).

${ }^{3}$ Teologo, PhD in Psicoanalisi Clinica. Ha lavorato per 15 anni con la Metodologia Scientifica (Metodo di Ricerca) nell'Orientamento alla Produzione Scientifica di studenti di Master e Dottorato di Ricerca. Specialista in Ricerche di Mercato e Ricerche focalizzate sull'area Salute.

${ }^{4}$ Biologo, Dottore di Ricerca in Malattie Tropiche, Professore e ricercatore del Corso di Educazione Fisica, Università Federale di Pará (UFPA).

${ }^{5}$ Biologo, Dottore di Ricerca in Teoria e Comportamento, Professore e ricercatore del Corso di Laurea in Chimica dell'Istituto di Educazione Di Base, Tecnica e Tecnologica di Amapá (IFAP) e del Corso di Laurea in Formazione Professionale e Tecnologica (PROFEPT IFAP).
}

RC: 73423

Disponível em: https://www.nucleodoconhecimento.com.br/formazione-it/tecnica-del- 
ago e fili, nonché immagini. L'uso della tecnica del ricamo libero - Le immagini descritte possono essere in grado di fornire agli studenti delle scuole superiori un maggiore sviluppo delle capacità di concentrazione (attenzione, attenzione), motricità e creatività. Anche la tecnica di rispettare l'individuo nella sua individualità di apprendimento e ludicity sembra influenzare l'aumento di queste capacità. La tecnica descritta può essere tranquillamente adattata a diverse regioni, come accade in Brasile, un paese di dimensioni continentali e diversità senza precedenti.

Parole chiave: Ricamo gratuito, Motricità, Concentrazione, Creatività.

\section{INTRODUZIONE}

All'interno del curriculum educativo brasiliano c'è una componente chiamata educazione artistica (BRASIL, 1997). L'educazione artistica è composta da diverse tecniche che portano allo sviluppo del pensiero logico, della creatività e della coordinazione motoria, utilizzando l'espressione del sentimento (EÇA, 2010). Tra queste tecniche ci sono opere manuali, cosiddette perché eseguite con le mani (RAMOS, 2013), come pittura, ricamo, uncinetto, macramê e artigianato (GUEDES et al., 2011).

È possibile produrre diversi tipi di lavoro manuale (GUEDES et al., 2011). Crafts è un gruppo di lavori manuali realizzati con diversi componenti creando le forme più varie (pittura, ricamo, uncinetto, macramê, basketry, ceramica) (RAMOS, 2013). Nella pittura, vengono solitamente utilizzate vernici o altre modalità di stampa e un substrato (carta, legno, tela, ecc.) per fare la rappresentazione grafica di un'immagine (CASTILHO et al., 2017). Nel ricamo un tessuto viene utilizzato come base dove l'insieme di diversi aghi e fili esegue finiture con forme o figure geometriche (RAMOS, 2013).

Nelle scuole elementari e superiori sembra più che appropriato l'insegnamento del lavoro manuale. L'artigianato lavora nell'interdisciplinarietà, un concetto importante

RC: 73423

Disponível em: https://www.nucleodoconhecimento.com.br/formazione-it/tecnica-del- 
per l'acquisizione globale di conoscenze. Stimola anche la creatività e la risoluzione dei problemi. Ciò si traduce in un cittadino umanizzato che comprende meglio l'altro e il mondo (TEIXEIRA, 2020).

Esistono diversi tipi di lavoro manuale che possono essere utilizzati nell'insegnamento. Tra questi, ricami a punto croce, uncinetto e maglieria sono lavori a mano che utilizzano linee di vario tipo intrecciate con aghi diversi e vengono insegnate fin dall'infanzia in varie regioni del Brasile (ALMEIDA, 2003; SANTOS et al., 2012). II macramê utilizza diversi tipi di filati intrecciati (sisal, corda) per oggetti di diverse forme (supporti di vasi, tende, abbellimenti) (VERMA et al., 2019).

Per sviluppare queste opere ci sono diverse tecniche di ricamo. Tra questi la tecnica del ricamo libero dove ogni persona borda secondo la propria creatività, senza parametri prestabiliti (MENDONÇA e AGUIAR, 2015).

Il lavoro manuale, come il ricamo, ad esempio, stimola la capacità motoria dei bambini facendoli utilizzare la muscolatura nei movimenti chiamati fine. La concentrazione è amplificata anche negli studenti con questa pratica scolastica. (TEIXEIRA, 2018).

II lavoro online stimola e aumenta la creatività negli studenti. L'espressione personale in cui si intrecciano la riproduzione delle immagini e la creazione stimola sia la conoscenza che la creazione stessa (BERGAMO e SILVA, 2020)

\section{GOL}

Descrivi una tecnica di ricamo in linea per lo sviluppo creativo e il motore applicabile nelle lezioni di educazione artistica delle scuole superiori.

RC: 73423

Disponível em: https://www.nucleodoconhecimento.com.br/formazione-it/tecnica-del- 


\section{MATERIALE E METODO}

\section{MATERIALI}

- Tessuto di cotone $50 \mathrm{~cm}$ x 50 centimetri, uno per studente;

- Cremagliera in legno di 30 centimetri, una per studente;

- Varie linee colorate, 08 metri per studente;

- Linee di matasse colorate varie, 08 metri per studente;

- Aghi per numeri di ricamo 22 e 24 per punto croce, un paio per ogni studente;

- Tagliare le forbici, una per ogni studente;

- Selezionare due (varie) immagini per studente e stamparle in bianco e nero di dimensioni 14 centimetri per 10 centimetri ( $1 / 4$ di carta A4).

\section{METODO}

Tecnica di ricamo gratuito - Immagini

Questa tecnica viene applicata e rifinita in quattro classi di 50 minuti ciascuna. Le lezioni devono avere un numero massimo di 10 studenti.

I materiali elencati devono essere disposti davanti a ogni studente all'inizio della lezione. Le immagini stampate devono essere posizionate in coppie casuali insieme al resto dei materiali. Sia le immagini che le linee possono essere scambiate tra gli studenti. Le immagini solo all'inizio dell'applicazione della tecnica e le linee in qualsiasi momento durante le lezioni.

RC: 73423

Disponível em: https://www.nucleodoconhecimento.com.br/formazione-it/tecnica-del- 
Passo 1: L'immagine su cui lavorare deve essere scelta da ogni studente, tra le immagini disponibili.

Fase 2: Ogni tessuto di cotone $(50 \times 50 \mathrm{~cm})$ deve essere posizionato nel rack di legno $(30 \mathrm{~cm})$, lasciandolo il più allungato possibile (Figura 1$)$.

Figura 1 Mostra il rack (A), il tessuto di cotone $(B)$ e il tessuto all'interno del rack (C).
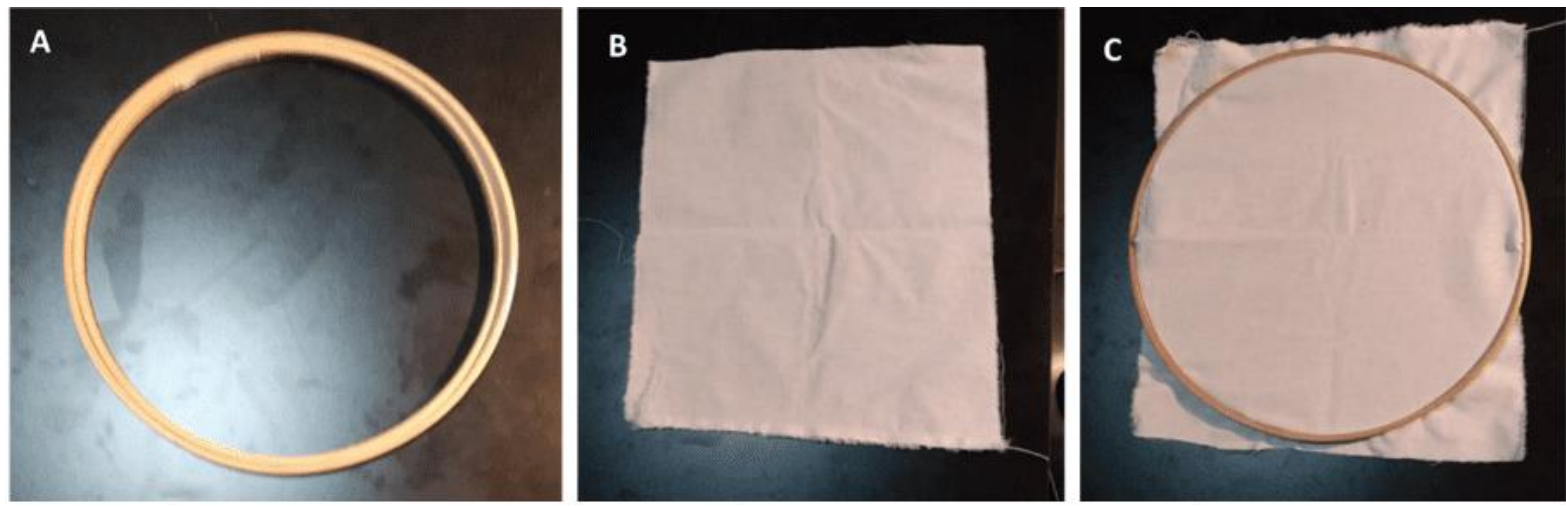

Passaggio 3: Gli studenti devono selezionare i colori e i tipi di linea (che possono essere entrambi i tipi) da utilizzare. Queste linee possono essere socializzate durante il processo (figura 2).

RC: 73423

Disponível em: https://www.nucleodoconhecimento.com.br/formazione-it/tecnica-del$\underline{\text { ricamo }}$ 
Figura 2 Mostra esempi di fili di cotone che possono essere utilizzati.

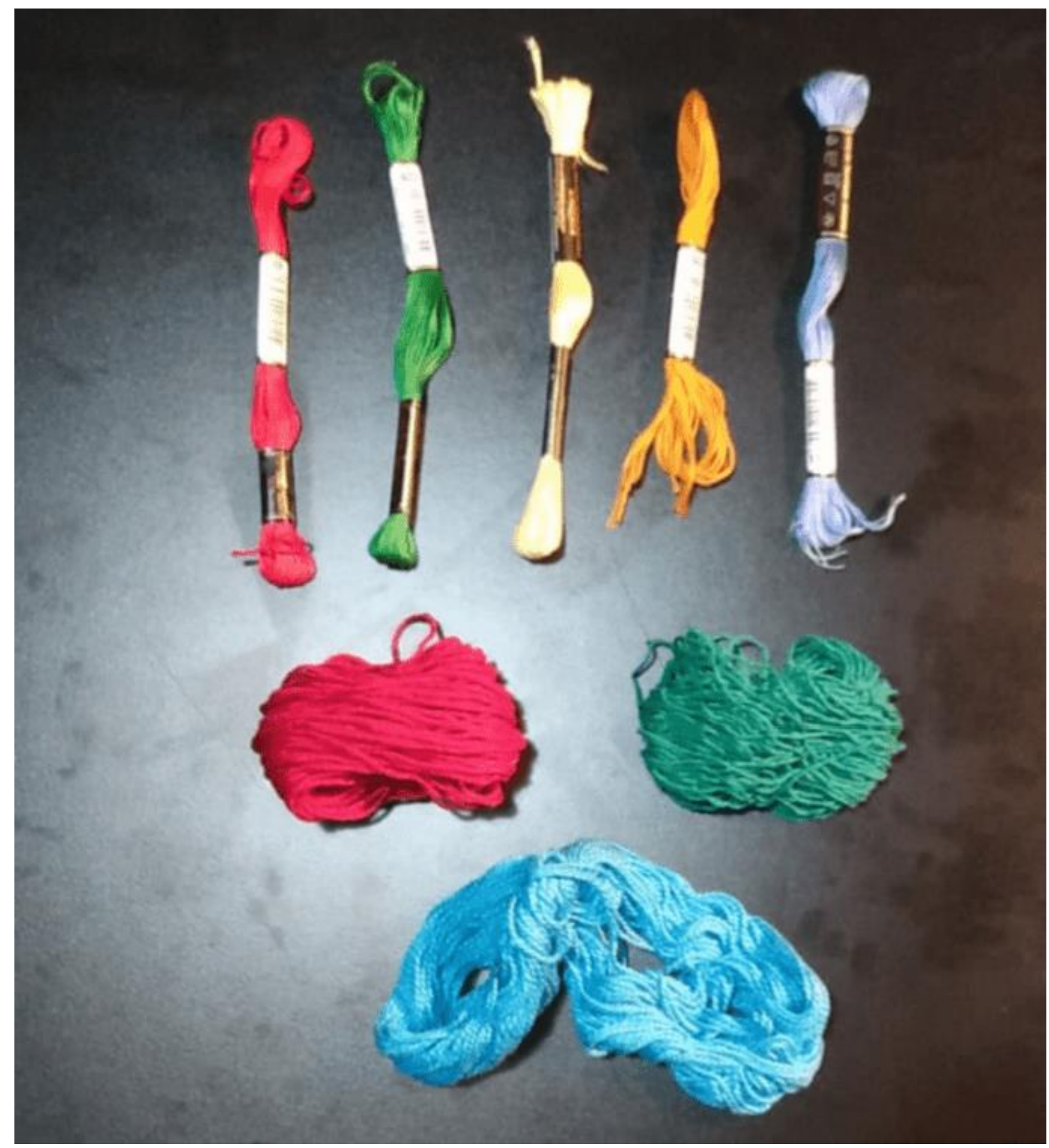

RC: 73423

Disponível em: https://www.nucleodoconhecimento.com.br/formazione-it/tecnica-delricamo 
Passo 4: Ogni studente taglia pezzi di $40 \mathrm{~cm}$ delle linee (tipo e colore) scelte per eseguire l'opera. Le linee possono essere commutate o meno durante il processo.

Passo 5: Ogni studente sceglie tra gli aghi da ricamo disponibili (22 o 24) e inserisce la prima delle linee in questo (Figura 3).

Figura 3 Mostra i numeri $22(\mathrm{~A})$ e $24(\mathrm{~B})$

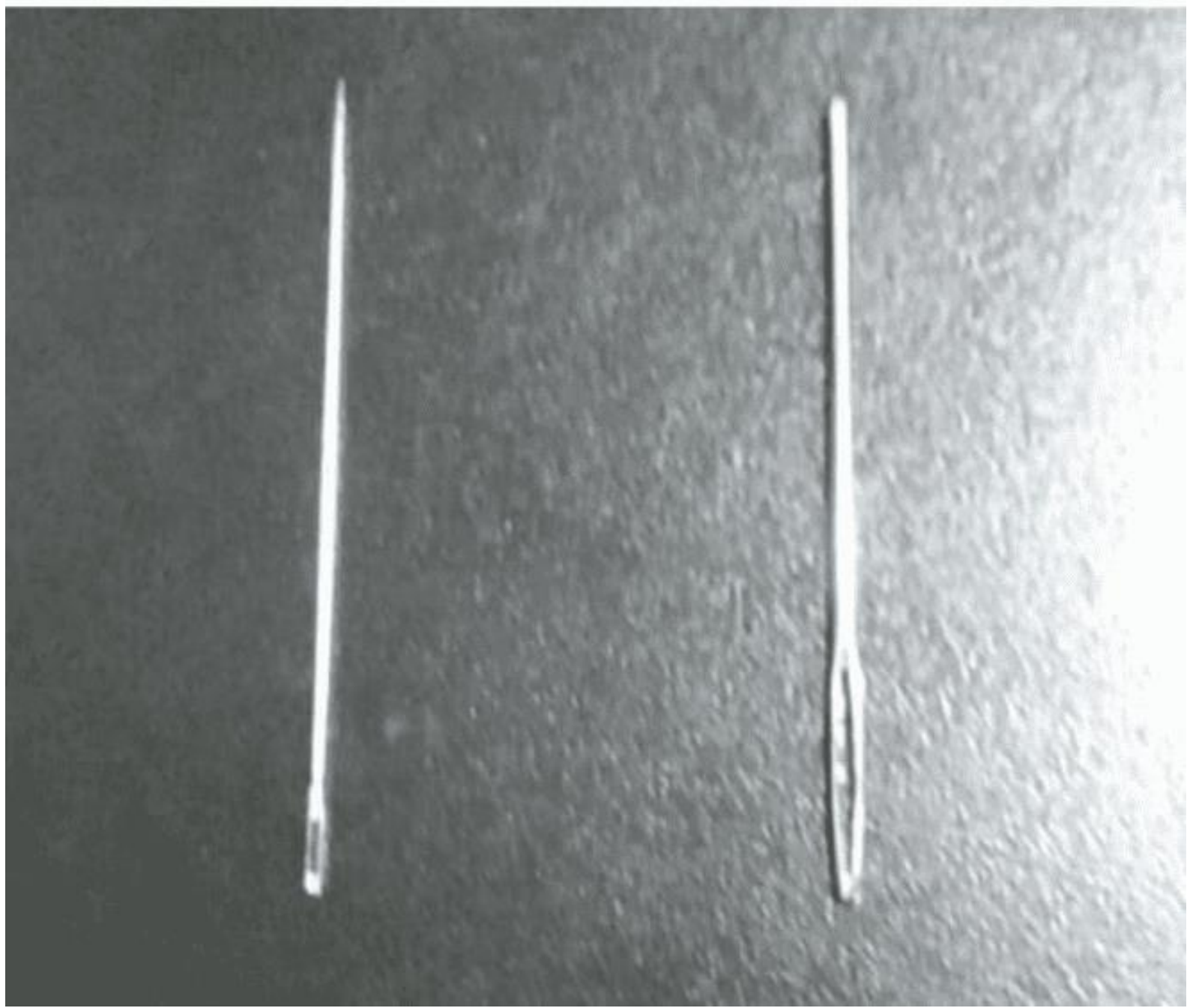

Passo 6: L'insegnante esemplifica in un tessuto, posto in un backstage, il punto chiamato "Dietro". Questo punto sarà realizzato in grandi dimensioni e con linea

RC: 73423

Disponível em: https://www.nucleodoconhecimento.com.br/formazione-it/tecnica-delricamo 
rossa per una migliore visualizzazione. L'insegnante insegnerà agli studenti le possibili dimensioni di questo tipo di punto (Figura 4).

Figura 4 Mostra i passaggi per realizzare il punto "Back", con filo di tessuto di cotone attaccato al rack di legno.
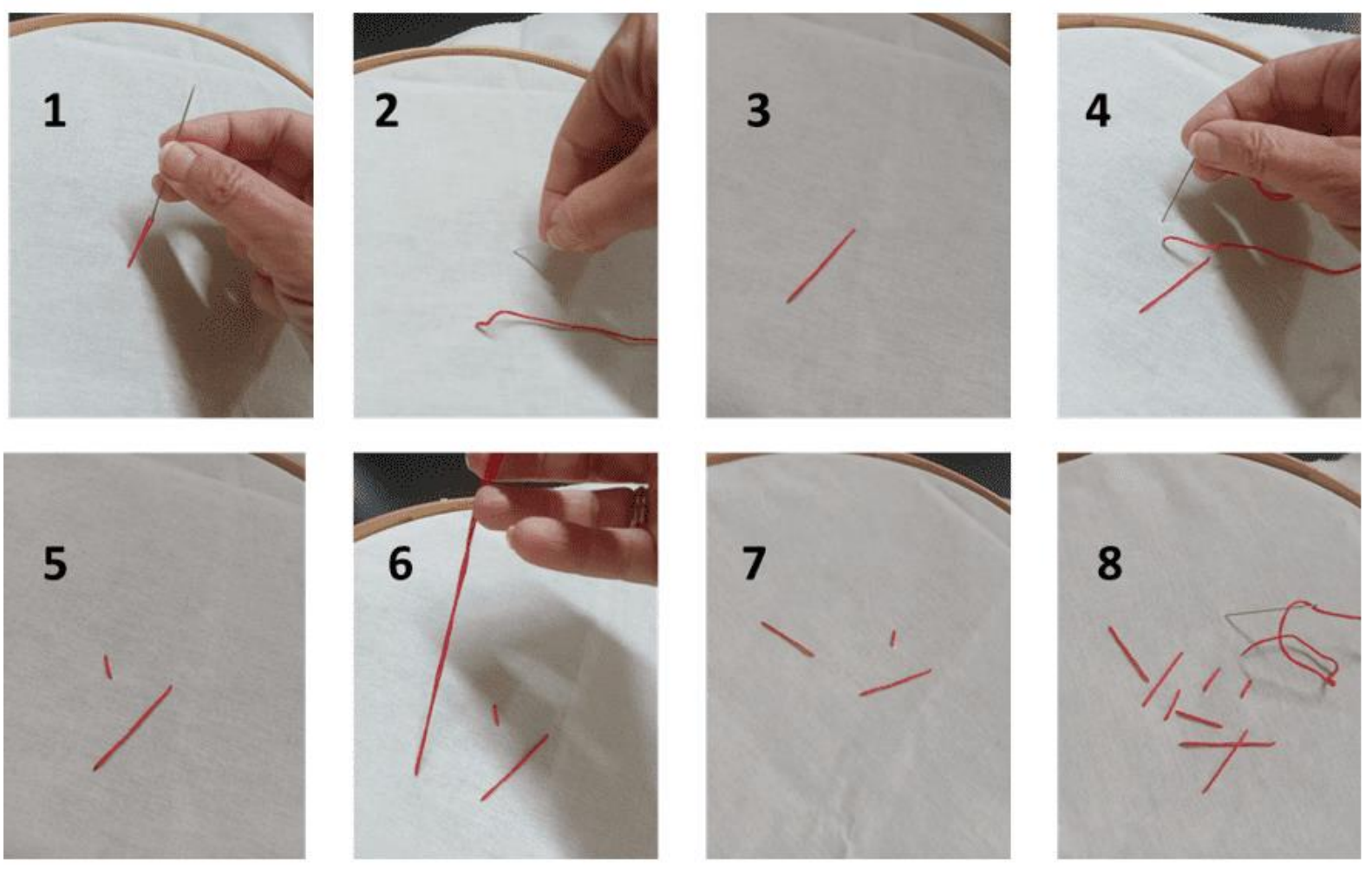

Passo 7: Ogni studente dovrebbe mettere di fronte a sé l'immagine che ha scelto in precedenza.

Passo 8: Dall'osservazione dell'immagine ogni studente dovrebbe cercare di riprodurre l'immagine o una libera interpretazione di essa nel tessuto, utilizzando il tipo di punto "Dietro". Dovrebbe anche scegliere le diverse dimensioni dei punti, usando una singola dimensione o alternandole.

RC: 73423

Disponível em: https://www.nucleodoconhecimento.com.br/formazione-it/tecnica-delricamo 


\section{RISULTATI ATTESI}

Si prevede che, alla fine del periodo, ogni studente finirà la propria rappresentazione con filo nel tessuto. Gli studenti dovrebbero anche presentare l'evoluzione nella loro capacità di osservare (concentrazione, concentrazione) motore e creativo.

\section{DISCUSSIONE}

L'atto di insegnamento e apprendimento è diventato un percorso razionale e meccanico, concettuale, rinunciando a ciò che è soggettivo, giocoso ed estetico. Negli studenti adolescenti moderni, i fattori psicoemotivi sembrano influenzare la formazione dei pensieri e, quindi, l'apprendimento. La pedagogia di Waldorf sostiene quindi l'arricchimento dell'apprendimento (concentrazione) attraverso attività ed esperienze artistiche. I contenuti delle discipline sono legati ad una pratica (attività artigianali, ad esempio) e ad un'attività artistica (chiamata veicolo didattico) (SILVA, 2015).

In una scuola con la filosofia Waldorf, insegni agli studenti il lavoro manuale. Gli adolescenti del decimo anno, secondo il curriculum scolastico, sono in grado di progettare oggetti fatti a mano utilizzando tecniche arazzo. Ciò richiede le capacità motorie dello studente e un'alta concentrazione (LUZ, 2016).

Ci sono studenti che hanno una sindrome che riunisce deficit di attenzione, deficit motorio e deficit di percezione, chiamata sindrome MPD. La ricerca mostra che gli individui con difficoltà nei settori dell'attenzione (concentrazione, messa a fuoco), della motricità (grossolana e fine) e del linguaggio, quando esposti all'insegnamento che privilegia l'espressione della libertà e delle componenti artistiche, comprese le opere manuali, tendono a presentare miglioramenti nei loro dipinti. La concentrazione (attenzione), la motricità e l'interazione sociale sono migliorate (SOUSA, 2011).

RC: 73423

Disponível em: https://www.nucleodoconhecimento.com.br/formazione-it/tecnica-del- 
Un caso di studio monote soggetto condotto su un periodo di due anni ha dimostrato che l'essere umano sviluppa una coordinazione corpo a corpo, indispensabile per la scrittura. Questo tipo di coordinamento è lo stesso che viene acquisito e automatizzato quando si impara e si padroneggiano le tecniche di lavoro manuale con la linea, ad esempio. Per l'apprendimento e la padronanza di questa motricità è necessario praticare, come avviene nelle lezioni di arte (PATERNOST, 2000).

Gli studenti con difficoltà di scrittura (disgrafia) di solito hanno un'intelligenza normale o superiore agli standard. La difficoltà che presentano ostacola, tuttavia, il loro rendimento scolastico. II processo di utilizzo del lavoro manuale con questo studente (maglieria, uncinetto e tessitura), associato alla diversità degli spazi e alla libertà di immaginazione (metodo Waldorf), consente esperienze fisiche che aiutano nel miglioramento di questo quadro (FONSECA et al., 2020).

Un rapporto portoghese sull'insegnamento supervisionato, condotto con corsi di istruzione primaria e secondaria, dimostra che gli studenti possono sviluppare la loro creatività attraverso diverse tecniche, tra cui il lavoro manuale. Tra i tipi di lavoro appaiono le opere con linee (uncinetto e maglieria) (GOUVEIA, 2012).

Gli adolescenti con tempi di esposizione più lunghi alla vita virtuale hanno alcune carenze rispetto agli adolescenti che hanno tempi di esposizione più brevi. L'attività motoria è inferiore negli individui che sono strettamente legati alle attività con lo schermo rispetto a coloro che praticano attività fisiche (sport). II tempo trascorso sui giochi virtuali, ad esempio, può causare un calo della concentrazione legato al pensiero calmo e alla contemplazione. Anche le attività virtuali sembrano fornire un calo della creatività, a scapito di coloro che utilizzano attività manuali (SETZER, 2014).

RC: 73423

Disponível em: https://www.nucleodoconhecimento.com.br/formazione-it/tecnica-delricamo 


\section{CONCLUSIONE}

L'uso della tecnica del ricamo libero - Le immagini descritte possono essere in grado di fornire agli studenti delle scuole superiori un maggiore sviluppo delle capacità di concentrazione (attenzione, attenzione), motricità e creatività.

Anche la tecnica di rispettare l'individuo nella sua individualità di apprendimento e ludicity sembra influenzare l'aumento di queste capacità.

La tecnica descritta può essere tranquillamente adattata a diverse regioni, come accade in Brasile, un paese di dimensioni continentali e diversità senza precedenti.

\section{RIFERIMENTI}

ALMEIDA, R. N. M. D. Plano de negócios de uma loja de artigos para tircô, crochê e bordado ponto cruz. 2003. 123p. (Graduação). Universidade Federal de Santa Catarina, Florianópolis SC.

BERGAMO, M. L.; SILVA, A. L. Doilies Digitais: Um Estudo da Aplicação de Criatividade Computacional ao Crochê. DATJournal, v. 5, n. 1, p. 138-152, 2020.

BRASIL. Parâmetros curriculares nacionais: arte / Secretaria de Educação Fundamental. SEF. Brasília DF: MEC/SEF: 130p. p. 1997.

CASTILHO, M. A. et al. Artesanato e saberes locais no contexto do desenvolvimento local. Interações (Campo Grande), v. 18, n. 3, 2017.

EÇA, T. T. A Educação Artística e as Prioridades Educativas do Início do Século XXI. Revista Iberoamericana de Educación, n. 52, p. 127-146, 2010.

RC: 73423

Disponível em: https://www.nucleodoconhecimento.com.br/formazione-it/tecnica-delricamo 
FONSECA, S. M. D. F. P. D. et al. Coordenação motora fina: do Direito às propostas pedagógicas da Pedagogia Waldorf em disgráficos. Braz. J. of Develop., v. 6, n. 9, p. 71688-71705, 2020.

GOUVEIA, L. R. D. R. Relatório da Prática de Ensino Supervisionada em Ensino de Artes Visuais no 3 Ciclo do Ensino Básico e Secundário. Universidade de Évora. Evora PT, p.1-112. 2012

GUeDES, M. H. M.; GUeDES, H. M.; ALMEIDA, M. E. F. Efeito da prática de trabalhos manuais sobre a autoimagem de idosos. Rev. bras. geriatr. gerontol., v. 14, n. 4, 2011.

LUZ, I. R. P. VIVÊNCIA DA TECELAGEM MANUAL EM UMA TURMA DE 8 ANO DA ESCOLA MUNICIPAL OSÓRIO ALEIXO DA SILVA. $2016.85 p$. (Especialização). Escola de Belas Artes da UFMG Universidade Federal de Minas Gerais, Belo Borizonte MG.

MENDONÇA, M. D.; AGUIAR, S. M. Linhas, Laços e Tramas na Terceira Idade. p. 15p., $2015.2 \quad$ Disponível em: < http://www.cp2.g12.br/blog/propgpec/files/2019/11/Linhas-La\%C3\%A7os-e-Tramasna-Terceira-Idade.pdf >. Acesso em: 21 jan 2021.

PATERNOST, V. A MOTRICIDADE COMO BASE PARA A APRENDIZAGEM: O Estudo de Um Caso pela Reorganização Neurofuncional 2000. 138p. (Mestrado). UNIVERSIDADE ESTADUAL DE CAMPINAS, Campinas SP.

RAMOS, S. P. Políticas e Processos Produtivos do Artesanato Brasileiro como Atrativo de um Turismo Cultura. Revista Rosa dos Ventos v. 5, n. 1, p. 44-59, 2013.

RC: 73423

Disponível em: https://www.nucleodoconhecimento.com.br/formazione-it/tecnica-delricamo 
SANTOS, D. B. S. D.; SILVA, F. S. D.; CARMO, G. D. Bordadeiras da Namoradinha do Sertão: estudo sobre a produção de bordados da cidade de São João dos Patos VII CONNEPI. Palmas TO 2012.

SETZER, V. W. EFEITOS NEGATIVOS DOS MEIOS ELETRÔNICOS EM CRIANÇAS, ADOLESCENTES E ADULTOS. Sao Paulo, 2014. Disponível em: < https://www.ime.usp.br/ vwsetzer/efeitos-negativos-meios.html >. Acesso em: 21 jan 2021.

SILVA, D. A. A. E. Educação e ludicidade: um diálogo com a Pedagogia Waldorf. Educar em Revista, n. 56, p. 101-113, 2015.

SOUSA, M. D. L. C. M. D. A aplicação da pedagogia Waldorf em alunos com síndrome de DAMP. 2011. 126p. (Mestrado). Universidade Católica Portuguesa, Viseu.

TEIXEIRA, I. A. Bordados em sala de aula. 2018. 45p. (Graduação). Universidade de Brasília, Brasília DF.

TEIXEIRA, J. V. S. A Educação Artística-uma prioridade no sistema educativo português: o contributo das expressões artísticas para o desenvolvimento integral do aluno no 1. CEB. 2020. 143 (Mestrado). Instituto Superior De Ciências Educativas Do Douro, Pènafiel.

VERMA, P.; MAHAJAN, S.; KAUR, D. Development of Macrame dresses using textile waste. International Journal of Education \& Management, v. 9, n. 3, p. 130133, 2019

Inviato: Gennaio 2021.

Approvato: Gennaio 2021

RC: 73423

Disponível em: https://www.nucleodoconhecimento.com.br/formazione-it/tecnica-del- 